1 Title: Biogeographical history shapes evolution of reproduction in a global warming scenario

4 Authors: Marta A. Santos ${ }^{1,2^{*}}$; Marta A. Antunes ${ }^{1,2}$; Afonso Grandela ${ }^{1,2}$; Ana Carromeu-

5 Santos $^{3}$; Ana S. Quina ${ }^{3}$; Mauro Santos ${ }^{1,4}$; Margarida Matos ${ }^{1,2 * *} \&$ Pedro Simões ${ }^{1,2^{* *}}$

\title{
Affiliations:
}

${ }^{1} \mathrm{cE} 3 \mathrm{c}$ - Centre for Ecology, Evolution and Environmental Changes \& CHANGE Global Change and Sustainability Institute, Lisboa, Portugal;

2 Departamento de Biologia Animal, Faculdade de Ciências, Universidade de Lisboa, Lisboa, Portugal;

${ }^{3}$ CESAM - Centre for Environmental and Marine Studies, Universidade de Aveiro and Faculdade de Ciências, Universidade de Lisboa, Lisboa, Portugal;

${ }^{4}$ Departament de Genètica i de Microbiologia, Grup de Genòmica, Bioinformàtica i Biologia Evolutiva (GBBE), Universitat Autònoma de Barcelona, Spain.

* - corresponding author, email: martasantos@fc.ul.pt, ORCID 0000-0003-1463-6037

** - joint last authors

6 Acknowledgments: The authors thank Inês Fragata for help in the graphic data 7 presentation. This study is financed by Portuguese National Funds through 'Fundação 8 para a Ciência e a Tecnologia' (FCT) within the projects PTDC/BIA-EVL/28298/2017 9 and cE3c Unit FCT funding project UIDB/00329/2020. PS and ASQ are funded by 10 national funds (OE), through FCT, in the scope of the framework contract foreseen in 11 the numbers 4, 5 and 6 of the article $23^{\text {rd }}$, of the Decree-Law 57/2016, of August 29, 12 changed by Law 57/2017, of July 19. MS is funded by grants CGL2017-89160P from 13 Ministerio de Economía y Competitividad (Spain; co-financed with the European Union 14 FEDER funds), and 2017SGR 1379 from Generalitat de Catalunya. 


\section{Biogeographical history shapes evolution of reproduction in a global}

\section{6 warming scenario}

\section{Abstract}

19 Adaptive evolution might be critical for animal populations to thrive on the fast-

20 changing natural environments. Ectotherms are particularly vulnerable to global

21 warming and, although their limited coping ability has been suggested, few real-time

22 evolution experiments have directly accessed their evolutionary potential. Here, we

23 report a long-term experimental evolution study addressing the evolution of thermal

24 reaction norms, after $\sim 30$ generations under different thermal environments. We

25 analyzed the evolutionary dynamics of Drosophila subobscura populations as a

26 function of the thermally variable environments in which they evolved and their distinct

27 biogeographical background. Our results showed clear differences between the

28 historically differentiated populations: while the northern $D$. subobscura populations

29 showed a temporal increase in performance at higher temperatures, their southern

30 counterparts presented the opposite pattern. This suggests that the northern populations

31 might be better equipped to cope with the current rising temperatures. Remarkably, no

32 effect of thermal selection was found. The lack of a clear long-term adaptive response at

33 higher temperatures after evolution under a global warming scenario raises concerns

34 about the evolutionary potential of ectotherms. Our results highlight the complex nature

35 of thermal responses in face of environmental heterogeneity and emphasize the

36 importance of considering intra-specific variation in thermal evolution studies.

37 Keywords: Drosophila, experimental evolution, global warming, historical

38 background, thermal adaptation, thermal reaction norms 


\section{Introduction}

Biodiversity is under great pressure due to rapid environmental changes

41 associated with global warming. Adaptive evolution and plasticity may be important

42 means for organisms to cope with the increasingly warmer temperatures (Chevin et al.

43 2010; Merilä et al. 2016). Plasticity can also be adaptive if, for instance, environmental

44 cues occurring during development allow for animals to better cope with environmental

45 conditions as adults (see reviews in (Kelly 2019; Rodrigues and Beldade 2020; Buckley

46 and Kingsolver 2021). Ectotherms are particularly vulnerable animals as their body

47 temperature is highly constrained by the environment, which has a major impact on

48 their biological processes and, thus, on their fitness (Angilletta 2009; Hoffmann and

49 Sgrò 2018). To make matters worse, recent evidence showed a limited ability of

50 ectotherms to evolutionarily respond to upper temperature shifts (Kellermann and van

51 Heerwaarden 2019; MacLean et al. 2019), as well as limited scope for plasticity in that

52 environmental context (Gunderson and Stillman 2015; Sørensen et al. 2016).

A thermal adaptive response will, expectedly, lead to an optimal performance

54 within the range of temperatures to which the organisms have most exposure, and lower

55 fitness at more extreme values (Angilletta 2009). In the context of global warming, one

56 might expect a shift in the optimum performance towards higher temperatures, provided

57 genetic variation is present and other evolutionary constraints do not play a major role

58 (Kellermann and van Heerwaarden 2019; Kristensen et al. 2020). These shifts can be

59 observed through changes in the thermal reaction norms, which describe the

60 population's performance as a function of temperature range. According to Angilletta et

61 al. (2003), different types of trade-offs may be involved in changes of elevation or

62 shape of thermal reaction norms. On the one hand, the allocation of resources (e.g.,

63 energy differently allocated to two traits) or acquisition trade-offs (e.g., between 
64 maximizing the acquisition of resources vs. minimizing the risk of mortality) will

65 mainly lead to shifts in trait means, i.e., to the elevation of the thermal reaction norm.

66 On the other hand, the evolution under a specialist-generalist trade-off will expectedly

67 lead to a change in shape, if an increase in performance at a given temperature causes a

68 decrease at other temperatures. These different sources of variation of thermal reaction

69 norms are not mutually exclusive. The genetic variation available for these different

70 dimensions will govern the evolution of reaction norms (Huey and Kingsolver 1989;

71 Angilletta et al. 2003; Berger et al. 2013). Generalist vs. specialist trade-offs can

72 underlie patterns of local adaptation, even though adaptation costs are not always

73 present (Hereford 2009). There is evidence of thermal plasticity suggestive of local

74 adaptation in natural populations of ectotherms, with populations geographically

75 distinct presenting different thermal reaction norms (Trotta et al. 2006; Berger et al.

76 2013; Austin and Moehring 2019; Klepsatel et al. 2019), but it is not universal (e.g.,

77 (Klepsatel et al. 2013; Clemson et al. 2016). It is, thus, critical to address how plasticity

78 evolves in face of rapid thermal changes and whether this variation is consistent across

79 populations, to better understand the consequences of the current global warming

80 scenario.

81 Experimental evolution, the real-time study of populations across several

82 generations, in highly controlled and reproducible conditions (Kawecki et al. 2012), is a

83 very powerful approach to address the adaptation to novel environmental conditions,

84 including the evolution of plasticity. It has been frequently used to characterize the

85 tempo and mode of thermal evolution of ectotherms, namely in ecological scenarios

86 associated with global warming (Schou et al. 2014; Manenti et al. 2015; Kellermann

87 and van Heerwaarden 2019; Kinzner et al. 2019; Liukkonen et al. 2021). The native

88 Palearctic Drosophila subobscura is an emblematic case study of ectotherm thermal 
adaptation. It shows local adaptation to distinct natural habitats and latitudinal clinal variation for chromosomal inversion frequencies in Europe, North America, and South America (Prevosti et al. 1988; Rezende et al. 2010). These karyotypic polymorphisms have been shown to change worldwide as a result of global warming (Balanyá et al. 2006) and to respond to sudden heat waves (Rodríguez-Trelles et al. 2013). Local adaptation in the reproductive performance of D. subobscura during heat stress has also been reported (Porcelli et al. 2017). This species presents clear plastic responses to thermal variation (Fragata et al. 2016), with a key role of developmental temperature in shaping adult reproductive performance (Simões et al. 2020; Santos et al. 2021a). The developmental temperature range of $D$. subobscura is $6-26^{\circ} \mathrm{C}$ (Moreteau et al. 1997; David et al. 2005; Schou et al. 2017) with optimal viability and preferred body temperature between $16^{\circ} \mathrm{C}$ and $20^{\circ} \mathrm{C}$ (Rego et al. 2010; Castañeda et al. 2013; Schou et al. 2017). Shifts in this species' thermal reaction norms have been observed for locomotor behavior (Mesas et al. 2021) and thermal tolerance (Schou et al. 2016; MacLean et al. 2019), although in the latter case mostly for lower thermal limits.

For the last three years, we have been performing a thermal experimental evolution study on historically differentiated $D$. subobscura populations, derived from the extreme latitudes of the European cline (Simões et al. 2017). These populations have been subjected to thermal selective regimes that differ in mean temperature and/or thermal amplitude (Santos et al. 2021b). We have previously found no evidence for evolutionary changes in thermal reaction norms after nine generations in circadian fluctuating or global warming-like environments (Santos et al. 2021b). This finding may result from a small number of elapsed generations or a general lack of genetic variation for response to thermal selection. 
Here, we present an extended report of the evolutionary response of these

114 populations, spanning $\sim 30$ generations of thermal evolution, and thus testing a longer-

115 term adaptive shift in thermal reaction norms. As in Santos et al. (2021), the thermal

116 reaction norms were estimated through the population's reproductive performance at

117 three different temperatures $\left(14^{\circ} \mathrm{C}, 18^{\circ} \mathrm{C}\right.$, and $\left.24^{\circ} \mathrm{C}\right)$. With this design, we aim to

118 respond to three main questions: (i) Does longer-term evolution under thermally diverse

119 environments change the populations' thermal reaction norm? (ii) Do thermal reaction

120 norms change between short- and longer-term evolution? (iii) Can we find the signature

121 of biogeographical history in the populations' thermal evolutionary response?

122 First and foremost, we expect that differential plasticity between thermal 123 selection regimes may arise in a longer-term study, provided there is a correlated change

124 in reaction norms due to adaptation to the dynamic thermal environments. Because of

125 almost 30 generations experiencing a wide range of temperatures, we foresee a higher

126 performance of the global warming-evolved populations in more extremal thermal

127 environments. Second, we expect that fluctuating and warming-evolved populations to

128 perform worse than the control lines in intermediate thermal conditions, assuming a

129 trade-off between maximal performance and performance breath of the reaction norms

130 (Huey and Kingsolver 1989; Angilletta et al. 2003). Finally, we think that geographical

131 variation for plasticity may become apparent, driven by an interaction of thermal

132 selection with the distinct genetic backgrounds.

\section{Material and Methods}

135 Population maintenance and thermal selection regimes 
137 of Drosophila subobscura in two contrasting latitudes of the species' European cline:

138 Adraga, Portugal $\left(38^{\circ} 48^{\prime} \mathrm{N}\right)$ and Groningen, The Netherlands $\left(53^{\circ} 13^{\prime} \mathrm{N}\right)$. The

139 laboratory populations (PT, from Portugal, and NL, from The Netherlands) were

140 established in early September 2013 and three-fold replicated shortly after. All $\mathrm{PT}_{1-3}$

141 and $\mathrm{NL}_{1-3}$ populations were maintained in discrete generations with synchronous 28-day

142 cycle, 12L:12D photoperiod, and constant $18^{\circ} \mathrm{C}$ temperature (control conditions, C).

143 The flies were reared in $\sim 30 \mathrm{~mm}^{3}$ glass vials with controlled densities in both eggs (70

144 eggs per vial) and adults (50 adults per vial). Egg collection for the following generation

145 was around peak fecundity (seven to ten days old flies) and the census sizes per

146 generation ranged between 500 and 1000 individuals (see (Simões et al. 2017), for more

147 details).

Two new thermal selection regimes were derived in January 2019, by the time

149 PT and NL populations had undergone 70 generations of lab evolution. The circadian

150 fluctuation regime ( $\mathrm{F}$ populations, $\mathrm{FPT}_{1-3}$ and $\mathrm{FNL}_{1-3}$ ) and the global warming regime

151 (W populations, $\mathrm{WPT}_{1-3}$ and $\mathrm{WNL}_{1-3}$ ) - see (Santos et al. 2021b). The F populations are

152 under a daily temperature regime that fluctuates between $15^{\circ} \mathrm{C}$ and $21^{\circ} \mathrm{C}$, with a mean

153 daily temperature of $18^{\circ} \mathrm{C}$, a cycle that is constant across generations. The W

154 populations experience a daily fluctuation initially similar to the $\mathrm{F}$ lines but with a per

155 generation increase of $0.18^{\circ} \mathrm{C}$ in daily mean and $0.54^{\circ} \mathrm{C}$ in daily amplitude (see the

156 thermal profiles on Figure 1). This simultaneous increase in thermal mean and

157 amplitude per generation was, to our knowledge, not yet tested in other experimental

158 studies. Taking into account the global warming pace of $0.1-0.3^{\circ} \mathrm{C}$ per decade (IPCC

159 2018), a mean increase of $0.18^{\circ} \mathrm{C}$ per generation is equivalent to the thermal changes

160 experienced in nature by an organism with a 10 -year generation time. The 
161

162

163

164

progressively divergent upper and lower temperature extremes (increases of $0.44^{\circ} \mathrm{C}$ in upper and $0.08^{\circ} \mathrm{C}$ in lower limits, per generation) fit well with the projected 2 -fold increase in thermal extremes relative to mean temperature in mid-latitude location (IPCC 2018).

The PT and NL populations have been kept at $18^{\circ} \mathrm{C}$ since their foundation and were already adapted to lab conditions when the two new thermal regimes were created, serving, thus, as controls. As they are expected to be at evolutionary equilibrium, they reflect the ancestral state prior to the start of the $\mathrm{F}$ and $\mathrm{W}$ selection regimes. All experimental populations were, otherwise, kept under the same conditions, as explained above. Census sizes were, in general, high for all thermal selection regimes with the exceptions referred below (see Figure S1).

The experimental populations were synchronously assayed twice: (1) after 9 generations of thermal evolution ( $\mathrm{F}$ and $\mathrm{W}$ ), when the control populations $(\mathrm{C})$ had 79 generations of lab adaptation; and (2) after 31 generations of $\mathrm{W}$ selection and 29 generations in the F regime, together with the controls (evolved for 99 generations in the standard lab conditions).

After 9 generations in the warming regime, the experimental populations had been subjected to temperatures ranging from $14.4^{\circ} \mathrm{C}$ to $24.5^{\circ} \mathrm{C}$, with a daily average of $19.4^{\circ} \mathrm{C}$ (Figure 1). By generation 22, the thermal mean and amplitude increases in the warming regime (peak temperature of $30.2^{\circ} \mathrm{C}$ ) had to be halted. Particularly low egg-toadult viability led to significant declines in population size mainly between generations 22 and 24 (see Figure S1). Since then, the warming populations have been kept in the temperature cycle of generation 20 , i.e., with a mean temperature of $21.4^{\circ} \mathrm{C}$, a lower extreme of $13.5^{\circ} \mathrm{C}$, and an upper extreme of $29.4^{\circ} \mathrm{C}$ (Figure 1). The temperature increase 
bioRxiv preprint doi: https://doi.org/10.1101/2022.02.25.481927; this version posted February 28, 2022. The copyright holder for this preprint (which was not certified by peer review) is the author/funder, who has granted bioRxiv a license to display the preprint in perpetuity. It is made available under aCC-BY-NC-ND 4.0 International license.

185 caused a progressively shorter development time (i.e., from egg to adult) and, by

186 generation 31 , had led to a $96 \mathrm{~h}$ reduction in the life-cycle length, that became 24 days. 
187

\section{Thermal plasticity assays}

To study the evolution of thermal plasticity, we tested the effect of different thermal environments on the reproduction of adult flies. We analyzed the reproductive performance of eighteen experimental populations (three thermal selection regimes $\mathrm{W}, \mathrm{F}$ and $\mathrm{C}$, two histories - NL and PT, and three replicate populations) subjected to three thermal treatments $\left(14^{\circ} \mathrm{C}, 18^{\circ} \mathrm{C}\right.$, and $\left.24^{\circ} \mathrm{C}\right)$ in two different time points - after nine and 31 generations of thermal evolution; these assays are thereon referred as G9 and G31, respectively. The results for the G9 assay were previously reported in (Santos et al. 2021b). Experimental protocols for both G9 and G31 assays were identical. Briefly, the temperature treatments involved a lifelong exposure to a constant temperature: colder $\left(14^{\circ} \mathrm{C}\right)$, intermediate $\left(18^{\circ} \mathrm{C}\right)$, or warmer $\left(24^{\circ} \mathrm{C}\right)$. These temperatures were chosen considering the range of viable developmental temperatures for D. subobscura (6 26 ${ }^{\circ}$, (Moreteau et al. 1997; David et al. 2005; Schou et al. 2016)). Maternal effects were minimized by rearing all populations in a common-garden environment, under control conditions $\left(18^{\circ} \mathrm{C}\right.$ and 28 -day life cycle), for one full generation, before each assay.

In each generation, sixteen pairs of virgin males and females, per population and treatment, were individually assayed with a total of 864 couples. The mating pairs were transferred to fresh medium every other day, for eight days, and the presence of eggs was checked daily. The eggs laid between days six and eight were counted under a stereoscope. Four life history traits were assessed: (1) age of first reproduction (number of days since emergence until the first laid egg), where lower values show faster maturity; (2) fecundity (total number of eggs laid between days six and eight), which addresses the females reproductive performance when the selective pressures are higher, i.e., near the age of egg collection for the following generation; (3) productivity (number 
212 of offspring obtained from the eggs of day eight), which conveys the females' ability to

213 produce viable progeny; and (4) juvenile viability (ratio between productivity and

214 fecundity at day eight), which measures developmental success. To obtain more reliable

215 estimates of viability, only vials with at least five eggs were considered for this trait,

216 with $\sim 9 \%$ of the total number of vials being excluded from this analysis. This was done

217 to adjust for the increase in fungal infections observed in vials with a very low number 218 of eggs.

Statistical methods

Raw data for the analyses is the mean value for each replicate population and 222 temperature combination (e.g., three data values for FPT populations in each of the 223 three temperature treatments). Data was analyzed by linear mixed effects models fitted 224 with REML (restricted maximum likelihood). Three factor ANOVAs (Type III Wald F 225 tests, Kenward-Roger degrees of freedom) were done to estimate significance levels of 226 differences between thermal selection regimes, histories and temperature treatments, as 227 well as their interactions. Homoscedasticity and normality were checked. Considering 228 the robustness of the analysis of variance, minor violations were accepted (Knief and 229 Forstmeier 2021). Arcsine transformation was applied to the juvenile viability data. All 230 statistical analyses were performed in R v4.0.4, using the lme4 (Bates et al. 2015), car 231 (Fox and Weisberg 2019), lawstat (Hui et al. 2008), emmeans and ggplot2 (Wickham 232 2016) packages. Two general models were used to analyze the evolution of thermal 233 reaction norms:

(1) $Y=\mu+$ History + Temp + Selection + Gen + AP\{History $\}+$ History $\times$ 235 Temp + History $\times$ Selection + Selection $\times$ Temp + History $\times$ Gen + Temp $\times$ Gen + 
Selection $\times$ Gen + Selection $\times$ History $\times$ Temp + History $\times$ Temp $\times$ Gen + History $\times$

Selection $\times$ Gen + Selection $\times$ Temp $\times$ Gen + Selection $\times$ History $\times$ Temp $\times$ Gen $+\varepsilon$

(2) $Y=\mu+$ History + Temp + Selection + Gen + Block + History $\times$ Temp +

History $\times$ Selection + Selection $\times$ Temp + History $\times$ Gen + Temp $\times$ Gen + Selection $\times$

Gen + Selection $\times$ History $\times$ Temp + History $\times$ Temp $\times$ Gen + History $\times$ Selection $\times$

Gen + Selection $\times$ Temp $\times$ Gen + Selection $\times$ History $\times$ Temp $\times$ Gen $+\varepsilon$

with $Y$ being the trait in study (age of first reproduction, fecundity, productivity, or viability); History, as the fixed factor corresponding to geographical origin (with PT and NL as categories); Selection as the fixed factor representing the thermal selection regimes (with three categories: Control, Warming, and Fluctuating); Temp as the fixed factor corresponding to the three temperature treatments; and Gen as the fixed factor with two categories (generations 9 and 31). In these models, interactions between Selection or History and Temperature test for differences in thermal plasticity due to those factors, while interactions between Generation and Temperature indicate temporal changes in plasticity between the two generations. In model (1), AP\{History $\}$ is the random effect consisting of the ancestral replicate population (i.e., $\mathrm{PT}_{1-3} ; \mathrm{NL}_{1-3}$ ) nested in History, from which the replicate populations of the three thermal selection regimes were originated (e.g., Ancestral $\mathrm{PT}_{1}$ generated Control $\mathrm{PT}_{1}$, Fluctuating $\mathrm{FPT}_{1}$ and Warming WPT $_{1}$ ). In model (2), Block was defined as the random effect, corresponding to the set of same-numbered replicate populations from all thermal regimes, assayed in the same pseudo-randomized experimental rack. For this analysis, we used as raw data in each generation the difference between the mean of each replicate population and the average of the three synchronously assayed control populations (e.g., $\mathrm{FPT}_{1}$-PT, with PT 
261

262

263

264

265

266

267

as average of $\left.\mathrm{PT}_{1-3}\right)$. As the control populations are long established in the laboratory and, thus, expectedly near evolutionary equilibrium, this procedure allows to remove environmental effects due to the between generation assay asynchrony (e.g., see (Fragata et al. 2016). In fact, analysis for the control raw data did not show significant variation between generations. Thus, the temporal analyses included standardized data for Warming and Fluctuating populations. Models including or excluding interactions with the random factors (Block or Ancestral Population) were tested and AIC was used to define the best model for each trait. For age of first reproduction and productivity, the model with Block as random effect without its interactions was used; the best suited model for fecundity and viability was the one with Ancestral Population as random effect without its interactions. For viability data analyses, both models (1) and (2) were tested including fecundity of day eight (F8) as a covariate (with models including and excluding its interactions with fixed factors), to account for variation in fecundity across the several thermal treatments. This analysis was also done separately for each selection regime $(\mathrm{W}$ and $\mathrm{F})$.

We addressed the evolutionary dynamics of thermal reaction norms by comparing longer (by generation 31) and shorter-term evolution data (by generation 9, published in (Santos et al. 2021b)). We analyzed differences in experimental populations associated with the thermal selective regime imposed (Selection, W vs. F), the geographical origin (History, PT vs. NL), the temperature treatments (Temp, $14^{\circ}$, $18^{\circ} \mathrm{C}$, and $24^{\circ} \mathrm{C}$ ), and the two assayed generations (Gen, 9 and 31 ).

Thermal reaction norms were also analyzed after 31 generations of thermal evolution (i.e., using only data from generation 31). The statistical models used were similar to (1) and (2), but without Generation as factor. Raw data for these analyses was the mean absolute value for each replicate population and temperature combination. 
286 Models including and excluding interactions with random effects were assessed with

287 AIC. Considering the traits A1R, fecundity and productivity the best model was model

288 (1) for fecundity and productivity, excluding interactions with the random effect AP;

289 model (2) for age of first reproduction, excluding interactions with the random effect

290 block. For this trait, the model with the lowest AIC was the one defining the random

291 effect AP and F8 as covariate without interactions with fixed or random factors.

293 Results

294 Evolutionary dynamics of thermal reaction norms

295 To address the dynamics of temporal changes in thermal reaction norms between 296 generation 31 and 9, we compared the population's reaction norm after 31 generations

297 of selection considering the three lifelong temperature treatments $\left(14^{\circ} \mathrm{C}, 18^{\circ} \mathrm{C}\right.$, and

$29824^{\circ} \mathrm{C}$ ), with the data after 9 generations (see (Santos et al. 2021b) and Figures 2 and 3).

299 First, we analyzed the combined effects of thermal selection (W vs. F), 300 biogeographical history and their interaction on the reaction norms' evolution for the 301 three lifelong temperatures in the two assayed generations (Table 1). No overall effects 302 of selection (Selection $x$ Gen) or its effects on the reaction norms (Selection $x$ Temp $x$ 303 Gen) across generations were observed. The only exception was a significant across304 generation difference in age of first reproduction between $\mathrm{W}$ and $\mathrm{F}$ lines, with an 305 increased performance at $18^{\circ} \mathrm{C}$ and $24^{\circ} \mathrm{C}$ in the warming populations (see Table $1, \mathrm{~A} 1 \mathrm{R}$, 306 Selection $x$ Generation; and Figures 2 and 3). Overall temporal changes between 307 populations with contrasting history were observed for age of first reproduction and 308 viability (see Table 1, History $x$ Gen). This effect resulted mainly from the temporal 309 pattern (better performance) of the northern populations in both selection regimes 
$310\left(\mathrm{FNL}_{1-3}\right.$ and $\left.\mathrm{WNL}_{1-3}\right)$ compared to the southern populations (see Figures 2 and 3).

311 Changes in reaction norms associated with biogeographical history were found for

312 productivity and viability (see Table 1, History $x$ Temp); however, the patterns of

313 variation differed between generations, leading to a significant History $x$ Temp $x$ Gen

314 interaction term (see below). Hence, the interpretation of this History x Temp interaction

315 should be cautious. In terms of the thermal reaction norm's evolution, all traits but age

316 of first reproduction showed significant temporal changes due to different geographical

317 histories (see Table 1, History $x$ Temp $x$ Gen). This variation was mostly due to the

318 relative performance of southern and northern populations at higher temperatures and

319 across generations (see Figures 2 and 3). After 31 generations of thermal selection, the

320 southern populations showed a general drop in performance at $24^{\circ} \mathrm{C}$, when compared to

321 generation 9, while the northern populations not only maintain their performance but

322 also had an increase in productivity. Given the effect of geographical history on the

323 evolution of thermal reaction norms, additional temporal analyses were performed on

324 each set of populations from the same geographical origin (e.g., WNL and FNL), see

325 Figures S2 and S3. Temporal changes in thermal reaction norms were significant for

326 productivity in both sets of geographical populations (Temp $x$ Gen; northern: $\mathrm{F}_{2,22}=5.64$,

$327 \mathrm{p}<0.011$; southern: $\left.\mathrm{F}_{2,22}=8.66, \mathrm{p}<0.002\right)$, and for fecundity in the southern populations

328 (Temp x Gen; southern: $\mathrm{F}_{2,22}=5.54, \mathrm{p}<0.012$; northern: $\mathrm{F}_{2,22}=1.27, \mathrm{p}>0.29$ ). However, no

329 significant temporal changes in reaction norms of populations from different thermal

330 selective regimes were found for any trait (Selection $x$ Temp $x$ Gen; $\mathrm{p}>0.05$ in all

331 analyses). Finally, there were no temporal changes in the reaction norms for viability

332 either for the northern or southern populations (Temp x Gen; $\mathrm{p}>0.05$ ).

333 Second, we tested the evolutionary change of each thermal regime's reaction

334 norm between generations 9 and 31. We did not detect a significant effect of the 
335 intercept or significant temporal changes in the overall performance of any thermal

336 regime (Table S1, intercept, and Gen). A significant effect of temperature was observed

337 for age of first reproduction and productivity in the $\mathrm{W}$ populations (Table S1, Temp) but

338 no temporal changes in the reaction norms were observed for any trait (Table S1, Gen $x$

339 Temp). Also, historical variation did not present a significant effect either on overall

340 performance (Table S1, History) or across generations (Table S1, History $x$ Gen).

341 However, history had a significant effect on the overall shape of the reaction norms for

342 productivity, in both warming and fluctuating populations, but not for the other traits

343 (Table S1, History $x$ Temp). In addition, a significant History x Temp x Gen interaction

344 was observed for fecundity in the $\mathrm{F}$ populations and productivity in both $\mathrm{F}$ and $\mathrm{W}$

345 populations, indicating historical differences in the evolution of thermal reaction norms

346 (see Table S1 and Figures 2 and 3, see also below). This temporal analysis was also

347 done separately for each combination of Selection and History populations (i.e., WPT,

348 WNL, FPT, and FNL) - see Table S2. Temporal changes in thermal reaction norms

349 (Table S2, Temp $x$ Gen) were observed for fecundity and productivity in the FPT

350 populations, and productivity for the WNL populations. Trait changes in FPT

351 populations resulted from the temporal decline in performance at higher temperatures,

352 while a temporal increase in productivity was observed for the WNL populations. This

353 productivity increase occured at both lower (approaching control values) and higher

354 temperatures (diverging from control values) - see Figures 2 and 3. 
We first analyzed the differences in thermal reaction norms between each 359 selective regime, fluctuating or warming, and the controls (Figures 2 and 3, 360 respectively). Differences between temperature treatments were observed for all traits in 361 both F vs. C and W vs. C comparisons (significant Temp factor, Tables S3 and S4).

362 Different traits showed different thermal responses: both colder $\left(14^{\circ} \mathrm{C}\right)$ and warmer $363\left(24^{\circ} \mathrm{C}\right)$ temperatures caused a decline in fecundity and productivity, when compared to 364 control conditions $\left(18^{\circ} \mathrm{C}\right)$; age of first reproduction showed reduced performance at 365 lower but not at warmer temperatures, while viability showed the reverse pattern (lower 366 performance at the warmest temperature) - see Figures 2 and 3; Tables S3 and S4. No 367 overall effects of biogeographical history or thermal selection were observed for most 368 traits. The exception was for age of first reproduction, where W populations showed 369 significantly lower values (see Table S3 and Figure 3).

The reaction norms of populations from different selection regimes (F vs. C or 371 W vs. C) or from different biogeographical histories (Portugal vs. The Netherlands) 372 were also not significantly different (Selection $x$ Temp and History $x$ Temp interactions, 373 respectively, Tables S3 and S4). A significant triple interaction History $x$ Selection $x$ 374 Temp was observed for productivity in the F vs. C comparison (Table S4, Figure 2), 375 indicating that populations from distinct geographical origin respond differently to 376 thermal selection. This effect was mainly due to a lower performance at $24^{\circ} \mathrm{C}$ of the 377 southern populations subjected to the fluctuating regime (FPT). 379 varying thermal environments (fluctuating vs. warming). Like what was found above, 380 differences between temperature treatments were observed for all traits (Table S5, 381 Temp). Overall differences in performance between selective regimes were only found 382 for age of first reproduction (Table S5, Selection) while no differences were found in 
383 the reaction norms between $\mathrm{F}$ and $\mathrm{W}$ regimes for any trait (Table S5, Selection $x$ Temp).

384 Interestingly, differences in the reaction norms of populations with diverse

385 biogeographical histories were found for productivity, mostly due to a better

386 performance of northern populations at higher temperatures (Table S5, History $x$ Temp). 


\section{Discussion}

The temperature increase and fast-changing environmental conditions associated

389 with global warming impose great stress on ectothermic animals and adaptation can be

390 key for their survival. Here, we present a 31 generation-long experimental evolution

391 study addressing the evolutionary dynamics of thermal plasticity in populations of $D$.

392 subobscura. Two populations with distinct biogeographical histories (northern and

393 southern Europe) were subjected to different thermal selection environments (daily

394 fluctuating and global warming scenarios).

Historical differences lead to contrasting evolutionary dynamics of thermal

reaction norms

We found that the evolutionary dynamics of thermal reaction norms across 22 generations of evolution (generation 9 vs. 31) for several life history traits varied between populations of contrasting geographical origin. This temporal variation in

401 plasticity was mostly driven by a differential performance at higher temperatures, with 402 the southern populations decreasing their reproductive performance across generations, 403 while their northern counterparts presented the opposite pattern; this effect was more 404 evident in their progeny productivity (Figures 2 and 3, Table 1). Geographical variation 405 in the shape of thermal reaction norms has been found in some studies of thermal 406 response in ectotherms (e.g., (Trotta et al. 2006; Berger et al. 2013; Austin and 407 Moehring 2019; Klepsatel et al. 2019) but not in others (Klepsatel et al. 2013; Clemson 408 et al. 2016). Particularly in D. subobscura, variation in thermal reaction norms has been 409 observed in populations from Sweden and Spain, with northern populations performing 410 worse at higher temperatures (Porcelli et al. 2017). One could, thus, expect northern $D$. 
411 subobscura natural populations to exhibit a steeper evolutionary response to rising

412 temperatures, as these populations are likely further away from the new optimum in a

413 warming scenario - and assuming that all populations evolve towards the same adaptive

414 peak (Matos et al. 2002; Losos 2011). Prior to the establishment of the thermal regimes,

415 our northern populations did not show a worse performance at higher temperatures

416 when compared to their southern counterparts (Simões et al. 2020; Santos et al. 2021a).

417 However, after 9 generations of thermal selection, though there was no robust evidence

418 for historical effects, we detected a tendency for lower performance of the northern

419 populations at higher temperatures across traits (Santos et al. 2021b). In this sense, the

420 contrasting temporal dynamics observed between geographical populations at higher

421 temperatures agrees with these expectations. Nevertheless, and assuming convergence,

422 this variation in dynamics would lead to lower historical differences after 31 generations

423 of thermal evolution. On the contrary, we found that populations are diverging through

424 time, with the northern populations increasing their performance at higher temperatures

425 and southern ones decreasing theirs. This pattern is particularly evident for productivity.

426 This is an interesting finding as it suggests that, given enough time, populations

427 historically from cooler climates might better overcome the current thermal challenges.

428 The increased performance in the northern populations could result from a better

429 developmental acclimation to higher temperatures, as both developmental and adult

430 stages were subjected to the same temperature, which is worthy of further investigation.

431 The presence of high genomic differentiation between the southern and northern

432 populations at the start of our experiment might also play a role in the observed

433 patterns. This is corroborated by previous findings in other D. subobscura populations

434 that were founded from the same natural locations and still exhibit a high degree of

435 genomic differentiation, even after 50 generations of lab evolution (Seabra et al. 2018). 
436 Further studies are needed to address the relevant genomic variation between the

437 evolving populations, so that we understand the driving force behind the geographical

438 variation and the temporal increase in performance in the northern populations.

439 Our results suggest non-linear and complex evolutionary trajectories in which an

440 interplay between geographical backgrounds and the varying thermal environments has

441 an important role. The absence of clear geographical variation in our short term study

442 (Santos et al. 2021b) and the later emergence of such differences reinforces the need for

443 caution when interpreting and extrapolating short term evolution patterns to longer term

444 evolution.

446 Evolution under different thermal selection regimes does not lead to

447 different thermal reaction norms

448 Considering the higher thermal variation in the warming vs. the fluctuating

449 environment with much more pronounced thermal extremes, one could expect a higher

450 performance of the warming-evolved populations at higher and lower temperatures, as

451 well as a lower performance at intermediate ones. This expectation is based on a

452 possible trade-off between thermal breath and maximal performance (Huey and

453 Kingsolver 1993). However, we did not find differences in the evolution of thermal

454 reactions norms between the warming and fluctuating populations, despite their

455 geographical origin. It might be the case that enzymatic constraints limit the evolution

456 of improved performance at both extremes (Angilletta et al. 2003), which, in turn, might

457 hinder the evolution of a more generalist performance of the warming populations. In

458 fact, other experimental evolution studies have seldom verified the evolution of such

459 predicted phenotypes as a response to thermal environmental heterogeneity, including 
460 the evolution of thermal specialists vs. generalists (e.g., (Berger et al. 2014; Condon et

461 al. 2014; Manenti et al. 2015) but see (Le Vinh Thuy et al. 2016).

462

463 Can populations adapt to a global warming environment?

464 We did not find a clear long-term adaptive response to higher temperatures after

46531 generations of thermal selection under a warming scenario, despite the trend of

466 improved productivity (i.e., number of offspring) of the northern populations (see

467 Figure 3 and Tables S2 and S3). Other experimental evolution studies addressing

468 adaptation to cumulative higher temperatures have also failed to find a clear adaptive

469 response to these more stressful environments (e.g., (Schou et al. 2014; Kinzner et al.

470 2019). Constrained plastic response of physiological traits at high temperatures was also

471 observed in comparative studies across species (Gunderson and Stillman 2015; Schou et

472 al. 2016; Sørensen et al. 2016; MacLean et al. 2019). Several causes might contribute to

473 the absence of a clear adaptive response, which was particularly evident in our southern

474 populations. Evolutionary constraints, such as trade-offs or an overall low genetic

475 variation for the measured traits, might play an important role (Hoffmann et al. 2017;

476 Kristensen et al. 2020). Methodological issues, such as the decoupling between the 477 assay environments and the selection regimes (e.g., constant vs. fluctuating

478 temperatures, low vs. high density reproduction) might also contribute. Another possible

479 explanation is that a part of the population's response to the thermal conditions might be

480 environmentally-based, resulting, for instance, from epigenetic and/or maternal

481 environmental effects (e.g., (Hoffmann and Bridle 2021). That being true, such effects

482 would not be detected in our experimental setup as they could have been mitigated or

483 removed by the one-generation common garden protocol at $18^{\circ} \mathrm{C}$ imposed prior to the

484 plasticity assays. The impact of common garden procedures and the environment in 
485 which they occur is an issue worthy of further consideration and analysis, particularly in

486 the context of experimental evolution.

487 The selective pressure experienced by populations in the new thermal

488 environment is also an important point to consider. There were times during thermal

489 evolution when the selective pressures in the warming environment were most likely

490 very high, as there were severe drops in the W population sizes' due to extremely low

491 juvenile viability. Nevertheless, in more recent generations, the populations showed an

492 obvious improvement in their ability to endure the maintenance conditions, with

493 consistently higher census sizes (Figure S1). This makes us wonder why such an

494 apparent adaptive response is not clearly detected in our life-history assays. We believe

495 that this might stem from a decoupling between the test environment and the imposed

496 selection environment. However, even if adaptation to the different thermal conditions

497 occurred, it did not produce conspicuous changes in the thermal reaction norms. In

498 future experiments, it will be important to test the exact environment in which the

499 populations evolve (i.e., local adaptation assays), alongside with changes in the thermal

500 reaction norms measured at constant temperatures.

502 Final remarks

503 We, here, report differential plasticity during evolution under thermally varying

504 environments, driven by the contrasting biogeographical history of the evolving

505 populations. The northern European populations appear to be coping better with these

506 dynamic thermal environments, as they showed a temporal increase in performance at

507 higher temperatures, particularly in terms of productivity. Contrary to our expectations,

508 the evolutionary changes in thermal reaction norms did not lead to an across-trait 
509 increased performance at lower and higher temperatures after long-term thermal

510 selection. Remarkably, our study shows a high impact of the previous biogeographical

511 history in the population's thermal evolutionary response, even after tens of generations

512 evolving in the same environment. These results highlight the importance of extending

513 the evolutionary studies to populations from different geographical sources, which have

514 adapted to local environmental conditions, to make more accurately intraspecific

515 generalizations. Our findings reinforce the likely complex nature of thermal responses

516 and illustrate the hardships of predicting evolutionary responses to global warming. 
517

518

519

520

521

522

523

524

525

526

527

528

529

530

531

532

533

534

535

536

537

538

539

540

541

542

543

544

545

546

547

548

549

550

551

552

553

554

555

556

557

558

559

560

\section{References}

Angilletta, M. J. 2009. Thermal Adaptation - A Theoretical and Empirical Synthesis. Oxford University Press Inc., New York.

Angilletta, M. J., R. S. Wilson, C. A. Navas, and R. S. James. 2003. Tradeoffs and the evolution of thermal reaction norms. Trends Ecol. Evol. 18:234-240.

Austin, C. J., and A. J. Moehring. 2019. Local thermal adaptation detected during multiple life stages across populations of Drosophila melanogaster . J. Evol. Biol. $1-10$.

Balanyà, J., J. M. Oller, R. B. R. Huey, G. W. G. Gilchrist, and L. Serra. 2006. Global Genetic Change Tracks Global Climate Warming in Drosophila subobscura. Science 313:1773-1775.

Bates, D., M. Maechler, B. Bolker, and S. Walker. 2015. Fitting linear mixed-effects models using lme4. J. Stat. Softw. 67:1-48.

Berger, D., E. Postma, W. U. Blanckenhorn, and R. J. Walters. 2013. Quantitative genetic divergence and standing genetic (co)variance in thermal reaction norms along latitude. Evolution 67:2385-2399.

Berger, D., R. J. Walters, and W. U. Blanckenhorn. 2014. Experimental evolution for generalists and specialists reveals multivariate genetic constraints on thermal reaction norms. J. Evol. Biol. 27:1975-1989.

Buckley, L. B., and J. G. Kingsolver. 2021. Evolution of Thermal Sensitivity in Changing and Variable Climates. Annu. Rev. Ecol. Evol. Syst. 52:563-586.

Castañeda, L. E., J. Balanyà, E. L. Rezende, and M. Santos. 2013. Vanishing chromosomal inversion clines in drosophila subobscura from Chile: Is behavioral thermoregulation to blame? Am. Nat. 182:249-259.

Chevin, L. M., R. Lande, and G. M. Mace. 2010. Adaptation, plasticity, and extinction in a changing environment: Towards a predictive theory. PLoS Biol. 8.

Clemson, A. S., C. M. Sgrò, and M. Telonis-Scott. 2016. Thermal plasticity in Drosophila melanogaster populations from eastern Australia: quantitative traits to transcripts. J. Evol. Biol. 29:2447-2463.

Condon, C., B. S. Cooper, S. Yeaman, and M. J. Angilletta. 2014. Temporal variation favors the evolution of generalists in experimental populations of Drosophila melanogaster. Evolution. 68:720-728.

David, J. R., L. O. Araripe, M. Chakir, H. Legout, B. Lemos, G. Pétavy, C. Rohmer, D. Joly, and B. Moreteau. 2005. Male sterility at extreme temperatures: A significant but neglected phenomenon for understanding Drosophila climatic adaptations. J. Evol. Biol. 18:838-846.

Fox, J., and S. Weisberg. 2019. An \{R\} Companion to Applied Regression. Third Edit. SAGE, Thousand Oaks CA.

Fragata, I., M. Lopes-Cunha, M. Bárbaro, B. Kellen, M. Lima, G. S. Faria, S. G. Seabra, M. Santos, P. Simões, and M. Matos. 2016. Keeping your options open: Maintenance of thermal plasticity during adaptation to a stable environment. Evolution 70:195-206.

Gunderson, A. R., and J. H. Stillman. 2015. Plasticity in thermal tolerance has limited 

potential to buffer ectotherms from global warming. Proc. R. Soc. B Biol. Sci. 282. Hereford, J. 2009. A quantitative survey of local adaptation and fitness trade-offs. Am. Nat. 173:579-588.

Hoffmann, A. A., and J. Bridle. 2021. The dangers of irreversibility in an age of increased uncertainty: revisiting plasticity in invertebrates. Oikos. https://doi.org/10.1111/oik.08715.

Hoffmann, A. A., and C. M. Sgrò. 2018. Comparative studies of critical physiological limits and vulnerability to environmental extremes in small ectotherms: How much environmental control is needed? Integr. Zool. 13:355-371.

Hoffmann, A. A., C. M. Sgrò, and T. N. Kristensen. 2017. Revisiting Adaptive Potential, Population Size, and Conservation. Trends Ecol. Evol. 32:506-517. Elsevier Ltd.

Huey, R. B., and J. G. Kingsolver. 1993. Evolution of resistance to high temperature in ectotherms. Am. Nat. 142:21-46.

Huey, R. B., and J. G. Kingsolver. 1989. Evolution of thermal sensitivity of ectotherm performance. Trends Ecol. Evol. 4:131-135.

Hui, W., Y. R. Gel, and J. L. Gastwirth. 2008. Lawstat: An R package for law, public policy and biostatistics. J. Stat. Softw. 28.

IPCC. 2018. Summary for Policymakers. P. 32 in P. R. S. Masson-Delmotte, V., P. Zhai, H.-O. Pörtner, D. Roberts, J. Skea, M. I. G. A. Pirani, W. Moufouma-Okia, C. Péan, R. Pidcock, S. Connors, J.B.R. Matthews, Y. Chen, X. Zhou, and and T. W. E. Lonnoy, T. Maycock, M. Tignor, eds. Global Warming of $1.5^{\circ} \mathrm{C}$. An IPCC Special Report on the impacts of global warming of $1.5^{\circ} \mathrm{C}$ above pre-industrial levels and related global greenhouse gas emission pathways, in the context of strengthening the global response to eradicate poverty.

Kawecki, T. J., R. E. Lenski, D. Ebert, B. Hollis, I. Olivieri, and M. C. Whitlock. 2012. Experimental evolution. Trends Ecol. Evol. 27:547-560.

Kellermann, V., and B. van Heerwaarden. 2019. Terrestrial insects and climate change: adaptive responses in key traits. Physiol. Entomol. 44:99-115.

Kelly, M. 2019. Adaptation to climate change through genetic accommodation and assimilation of plastic phenotypes. Philos. Trans. R. Soc. B Biol. Sci. 374.

Kinzner, M. C., A. Gamisch, A. A. Hoffmann, B. Seifert, M. Haider, W. Arthofer, B. C. Schlick-Steiner, and F. M. Steiner. 2019. Major range loss predicted from lack of heat adaptability in an alpine Drosophila species. Sci. Total Environ. 695: 133753.

Klepsatel, P., M. Gáliková, N. De Maio, C. D. Huber, C. Schlötterer, and T. Flatt. 2013. Variation in thermal performance and reaction norms among populations of Drosophila melanogaster. Evolution 67:3573-3587.

Klepsatel, P., T. N. Girish, H. Dircksen, and M. Gáliková. 2019. Reproductive fitness of Drosophila is maximised by optimal developmental temperature. J. Exp. Biol. 222:1-11.

Knief, U., and W. Forstmeier. 2021. Violating the normality assumption may be the lesser of two evils. Behav. Res. Methods 53:2576-2590.

Kristensen, T. N., T. Ketola, and I. Kronholm. 2020. Adaptation to environmental stress at different timescales. Ann. N. Y. Acad. Sci. 1476:5-22. 
Le Vinh Thuy, J., J. M. VandenBrooks, and M. J. Angilletta. 2016. Developmental plasticity evolved according to specialist-generalist trade-offs in experimental populations of Drosophila melanogaster. Biol. Lett. 12.

Liukkonen, M., I. Kronholm, and T. Ketola. 2021. Evolutionary rescue at different rates of environmental change is affected by trade $\square$ offs between short $\square$ term performance and long $\square$ term survival. J. Evol. Biol. 34:1177-1184.

Losos, J. B. 2011. Convergence, adaptation, and constraint. Evolution. 65:1827-40.

MacLean, H. J., J. G. Sørensen, T. N. Kristensen, V. Loeschcke, K. Beedholm, V. Kellermann, and J. Overgaard. 2019. Evolution and plasticity of thermal performance: An analysis of variation in thermal tolerance and fitness in 22 Drosophila species. Philos. Trans. R. Soc. B Biol. Sci. 374.

Manenti, T., V. Loeschcke, N. N. Moghadam, and J. G. Sørensen. 2015. Phenotypic plasticity is not affected by experimental evolution in constant, predictable or unpredictable fluctuating thermal environments. J. Evol. Biol. 28:2078-2087.

Matos, M., T. Avelar, and M. R. Rose. 2002. Variation in the rate of convergent evolution $\square$ : adaptation to a laboratory environment in Drosophila subobscura. J. Evol. Biol. 15:673-682.

Merilä, J., and A. A. Hoffmann. 2016. Evolutionary Impacts of Climate Change. Oxford Res. Encycl. Environ. Sci., doi: 10.1093/acrefore/9780199389414.013.136.

Mesas, A., A. Jaramillo, and L. E. Castañeda. 2021. Experimental evolution on heat tolerance and thermal performance curves under contrasting thermal selection in Drosophila subobscura. J. Evol. Biol. 34:767-778.

Moreteau, B., J.-P. Morin, G. Pertavy, E. Pla, and J. R. David. 1997. Evolutionary changes of nonlinear reaction norms according to thermal adaptation $\square$ : a comparison of two Drosophila species Changements au tours de Evolution de normes de reaction. Acad. Sci. Paris 320:833-841.

Porcelli, D., K. J. Gaston, R. K. Butlin, and R. R. Snook. 2017. Local adaptation of reproductive performance during thermal stress. J. Evol. Biol. 30:422-429.

Prevosti, A., G. Ribo, L. Serra, M. Aguade, J. Balana, M. Monclus, and F. Mestres. 1988. Colonization of America by Drosophila subobscura: Experiment in natural populations that supports the adaptive role of chromosomal-inversion polymorphism. Proc. Natl. Acad. Sci. 85:5597-5600.

Rego, C., J. Balanyà, I. Fragata, M. Matos, E. L. Rezende, and M. Santos. 2010. Clinal patterns of chromosomal inversion polymorphisms in Drosophila subobscura are partly associated with thermal preferences and heat stress resistance. Evolution 64:385-397.

Rezende, E. E. L., J. Balanyà, F. Rodríguez-Trelles, C. Rego, I. Fragata, M. Matos, L. Serra, and M. Santos. 2010. Climate change and chromosomal inversions in Drosophila subobscura. Clim. Res. 43:103-114.

Rodrigues, Y. K., and P. Beldade. 2020. Thermal Plasticity in Insects' Response to Climate Change and to Multifactorial Environments. Front. Ecol. Evol. 8:1-12.

Rodríguez-Trelles, F., R. Tarrío, and M. Santos. 2013. Genome-wide evolutionary response to a heat wave in Drosophila. Biol. Lett. 9:20130228.

Santos, M. A., A. Carromeu-Santos, A. S. Quina, M. Santos, M. Matos, and P. Simões. 2021a. High developmental temperature leads to low reproduction despite adult 

temperature. J. Therm. Biol. 95:102794.

Santos, M. A., A. Carromeu-Santos, A. S. Quina, M. Santos, M. Matos, and P. Simões. 2021b. No evidence for short-term evolutionary response to a warming environment in Drosophila. Evolution 75:2816-2829.

Schou, M. F., T. N. Kristensen, V. Kellermann, C. Schlötterer, and V. Loeschcke. 2014. A Drosophila laboratory evolution experiment points to low evolutionary potential under increased temperatures likely to be experienced in the future. J. Evol. Biol. 27:1859-1868.

Schou, M. F., T. N. Kristensen, A. Pedersen, B. Göran Karlsson, V. Loeschcke, and A. Malmendal. 2017. Metabolic and functional characterization of effects of developmental temperature in Drosophila melanogaster. Am. J. Physiol. Regul. Integr. Comp. Physiol. 312:R211-R222.

Schou, M. F., M. B. Mouridsen, J. G. Sørensen, and V. Loeschcke. 2016. Linear reaction norms of thermal limits in Drosophila: Predictable plasticity in cold but not in heat tolerance. Funct. Ecol. 31:934-945.

Seabra, S. G., I. Fragata, M. A. Antunes, G. S. Faria, M. A. Santos, V. C. Sousa, P. Simões, and M. Matos. 2018. Different genomic changes underlie adaptive evolution in populations of contrasting history. Mol. Biol. Evol. 35:549-563.

Simões, P., I. Fragata, S. Seabra, G. S. Faria, M. A. Santos, M. R. Rose, M. Santos, and M. Matos. 2017. Predictable phenotypic, but not karyotypic, evolution of historically differentiated populations. Sci. Rep. 7:1-12.

Simões, P., M. A. Santos, A. Carromeu-Santos, A. S. Quina, M. Santos, and M. Matos. 2020. Beneficial developmental acclimation in reproductive performance under cold but not heat stress. J. Therm. Biol. 90:102580.

Sørensen, J. G., T. N. Kristensen, and J. Overgaard. 2016. Evolutionary and ecological patterns of thermal acclimation capacity in Drosophila: is it important for keeping up with climate change? Curr. Opin. Insect Sci. 17:98-104.

Trotta, V., F. C. F. Calboli, M. Ziosi, D. Guerra, M. C. Pezzoli, J. R. David, and S. Cavicchi. 2006. Thermal plasticity in Drosophila melanogaster: A comparison of geographic populations. BMC Evol. Biol. 6:1-13.

Wickham, H. 2016. ggplot2: Elegant Graphics for Data Analysis. Springer-Verlag New York. 


\section{Tables}

685 Table 1. Evolutionary dynamics of $\mathrm{W}$ and $\mathrm{F}$ populations. Data refers to average 686 population values standardized to the controls. The $\mathrm{F}$ statistic shows the degrees of 687 freedom of effect and error. Significance levels for $F$ values: p> 0.05 n.s.; $6880.05>\mathrm{p}>0.01 * ; 0.01>\mathrm{p}>0.001 * * ; \mathrm{p}<0.001 * * *$. Only the model parameters with a 689 significant effect in at least one trait are presented.

690

\begin{tabular}{ccccc}
\hline Model parameters & $\begin{array}{c}\text { Age of First } \\
\text { Reproduction (A1R) }\end{array}$ & Fecundity & Productivity & Viability \\
\hline History*Temp & $\mathrm{F}_{2,4}=2.300$ n.s. & $\mathrm{F}_{2,44}=1.111$ n.s. & $\mathrm{F}_{2,46}=7.250^{* *}$ & $\mathrm{~F}_{2,43.6}=3.594^{*}$ \\
History ${ }^{*} \mathrm{Gen}$ & $\mathrm{F}_{1,28}=11.08^{* *}$ & $\mathrm{~F}_{1,44}=0.007$ n.s. & $\mathrm{F}_{1,46}=2.236$ n.s. & $\mathrm{F}_{1,43.1}=4.613^{*}$ \\
Selection ${ }^{*} \mathrm{Gen}$ & $\mathrm{F}_{1,28}=7.185^{*}$ & $\mathrm{~F}_{1,44}=0.571$ n.s. & $\mathrm{F}_{1,46}=0.108$ n.s. & $\mathrm{F}_{1,43.0}=0.001$ n.s. \\
History${ }^{*}$ Temp $^{*}$ Gen & $\mathrm{F}_{2,28}=2.911$ n.s. & $\mathrm{F}_{2,44}=6.054^{* *}$ & $\mathrm{~F}_{2,46}=14.811^{* * *}$ & $\mathrm{~F}_{2,43.8}=5.459^{* *}$ \\
\hline
\end{tabular}

Figure Legends

Figure 1. Daily temperature profiles of the experimental regimes after nine (G9) and 696 thirty-one (G31) generations of thermal selection. The warming profile was kept unchanged from generation 20 on.

Figure 2. Evolution of the thermal reaction norms of the Fluctuating (F) and Control (C) thermal selection regimes after 9 and 31 generations of selection. Data show the average and $95 \%$ confidence intervals for each thermal regime at each time point, with average values of each replicate population as raw data. Viability data is arcsine transformed. A1R - Age of first reproduction.

Figure 3. Evolution of the thermal reaction norms of the Warming (W) and Control (C) thermal selection regimes after 9 and 31 generations of selection. Data show the average and $95 \%$ confidence intervals for each thermal regime at each time point, with average 708 values of each replicate population as raw data. Viability data is arcsine transformed. 709 A1R - Age of first reproduction. 
bioRxiv preprint doi: https://doi.org/10.1101/2022.02.25.481927; this version posted February 28, 2022. The copyright holder for this preprint (which was not certified by peer review) is the author/funder, who has granted bioRxiv a license to display the preprint in perpetuity. It is made available under aCC-BY-NC-ND 4.0 International license.

\section{$711 \quad$ Figures}

712

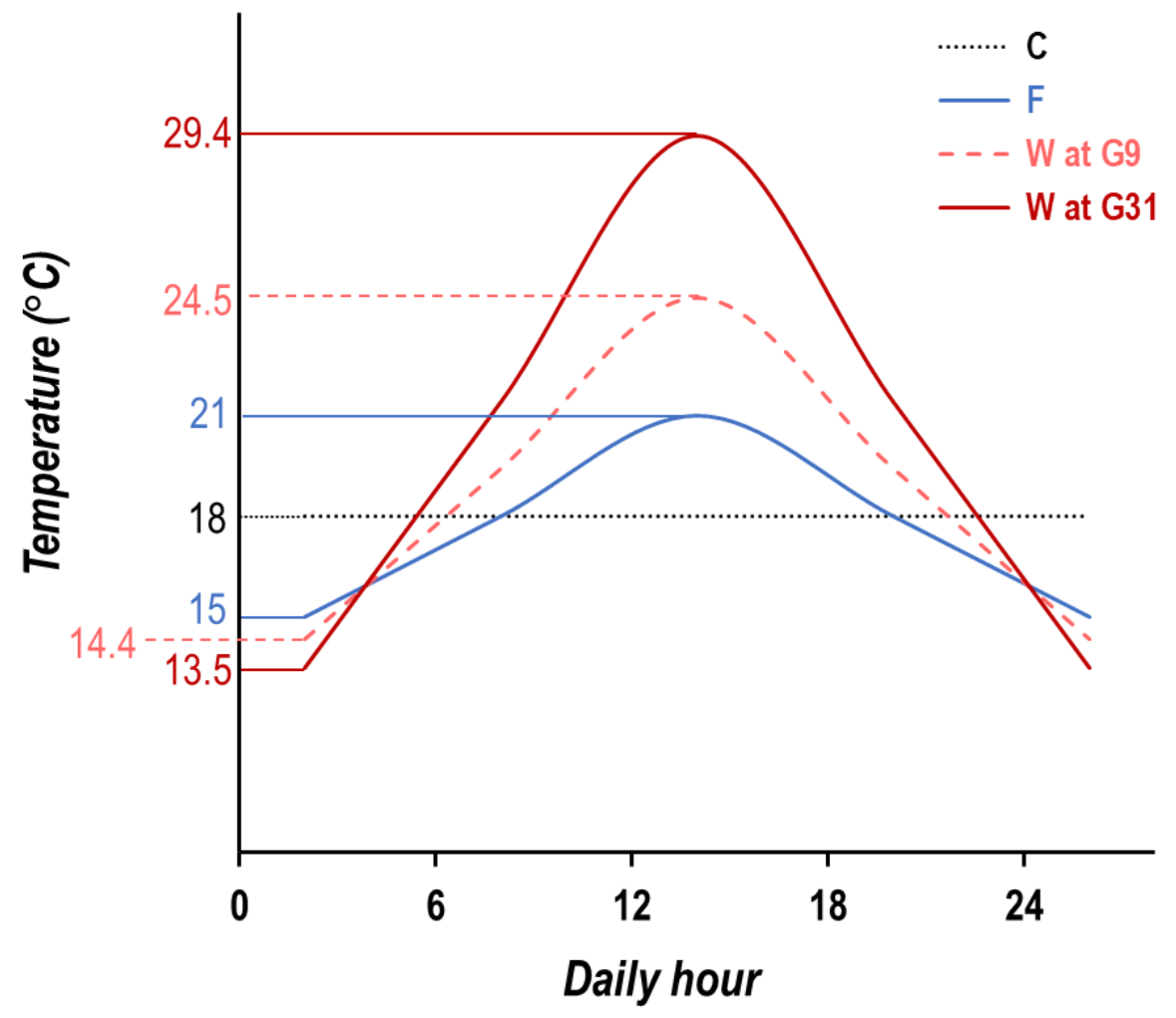

$714 \quad$ Figure 1 
Thermal Performance Curves in F vs. C populations
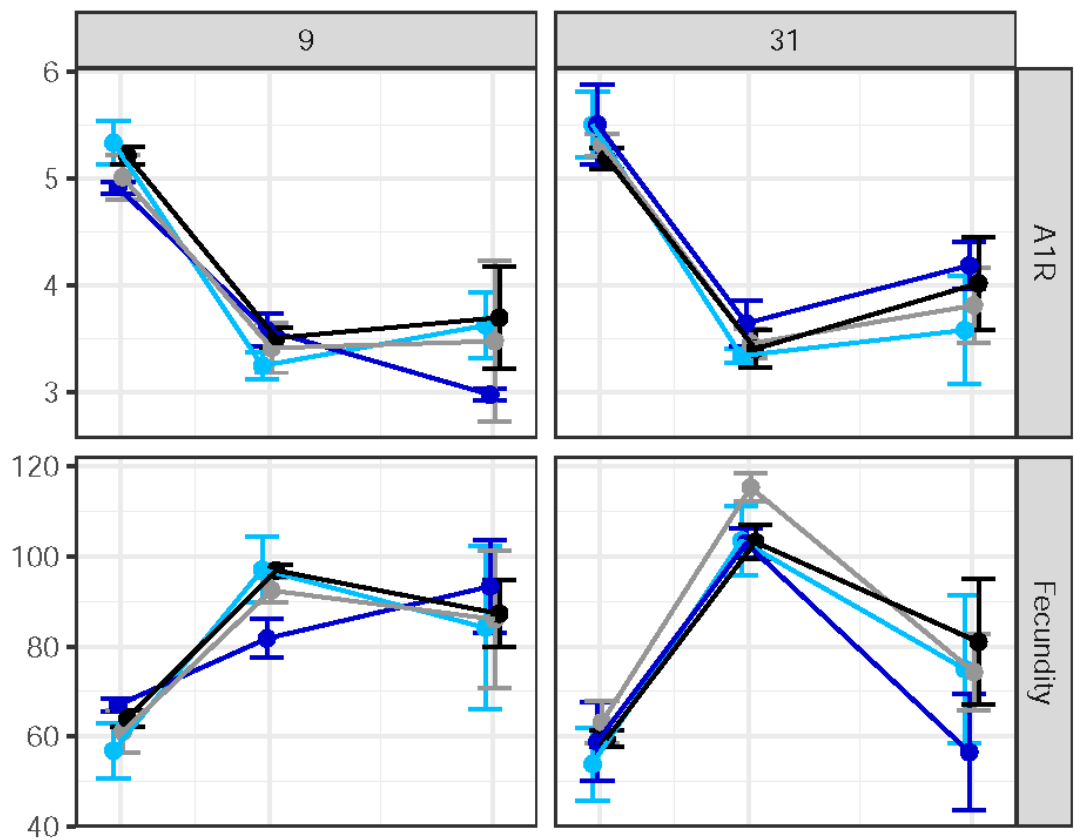

Population
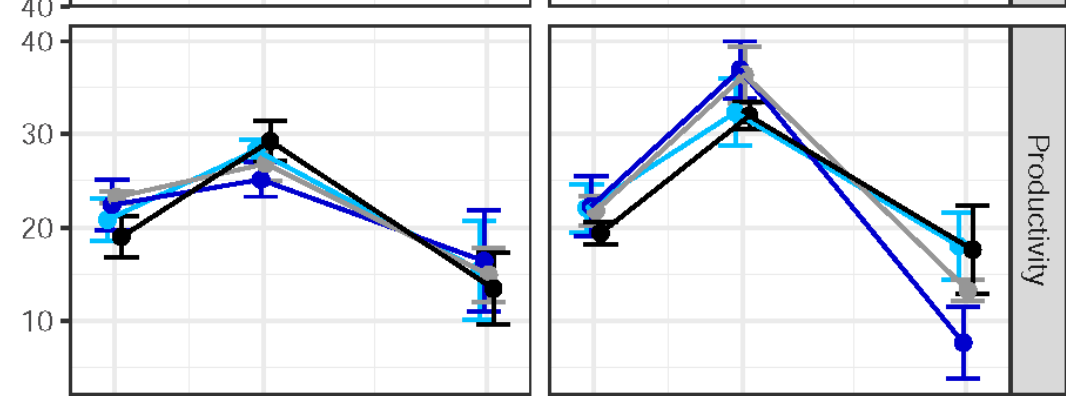

$\rightarrow-F N L$

$\rightarrow$ FPT

$-\mathrm{NL}$

$\rightarrow \mathrm{PT}$
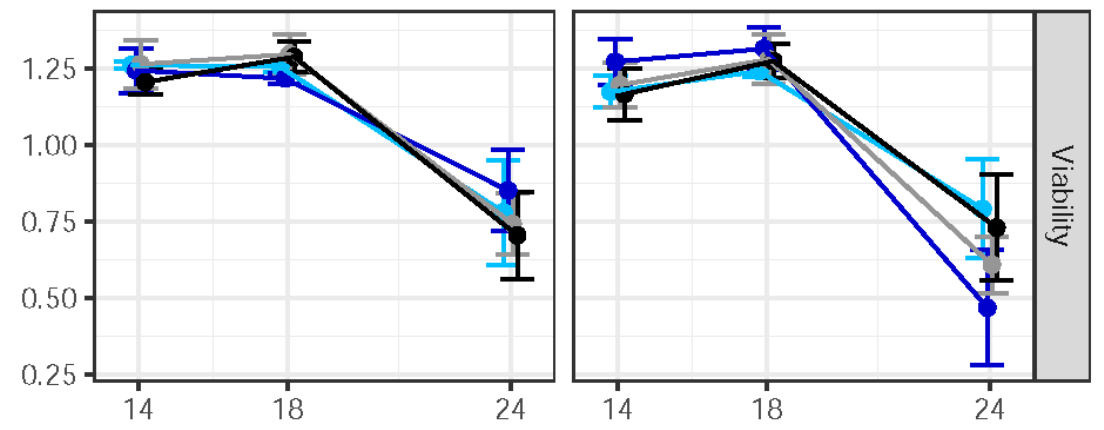

Temperature $\left({ }^{\circ} \mathrm{C}\right)$

716 Figure 2 
bioRxiv preprint doi: httos://doi org/10 1101/2022 02 25 481927: this version posted February 28,2022 The copvriaht holder for this preprint (which was not certified by peer review) is the author/funder, who has granted bioRxiv a license to display the preprint in perpetuity. It is made available under aCC-BY-NC-ND 4.0 International license.

Thermal Performance Curves in W vs. C populations
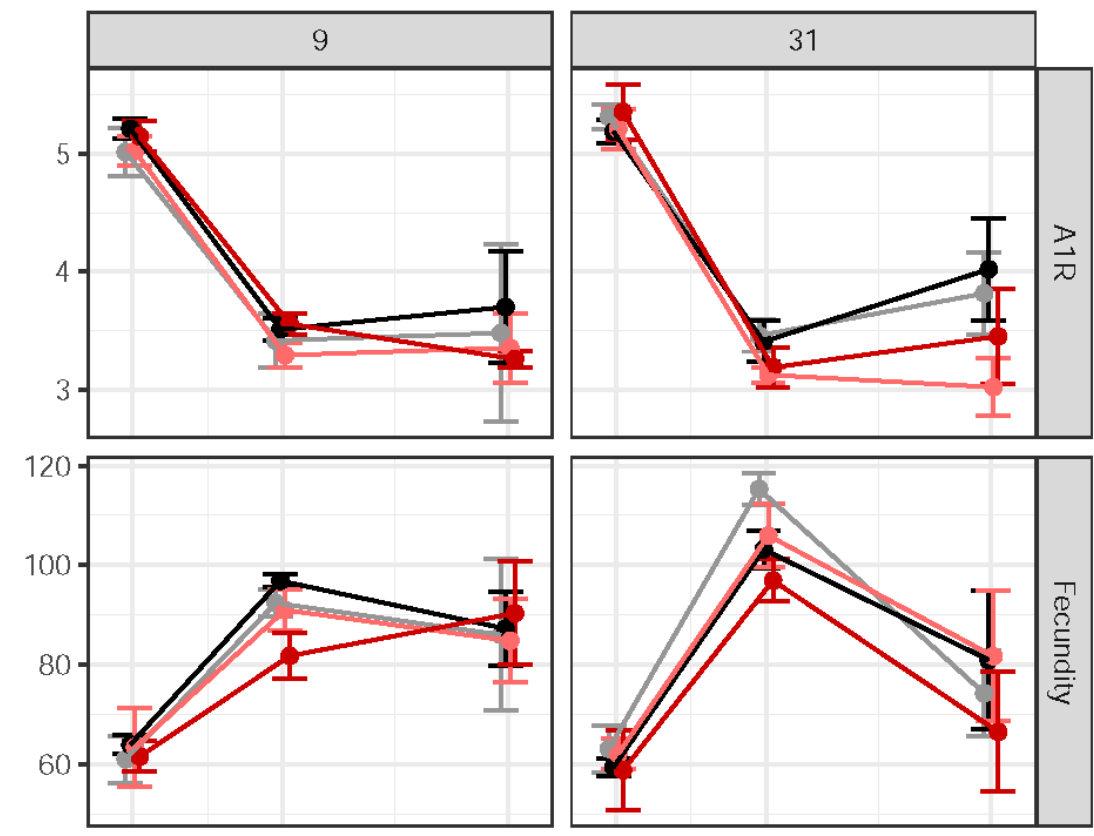

Population

$\rightarrow$ NL
$\rightarrow$ PT
$\rightarrow$ WNL
$\rightarrow$ WPT
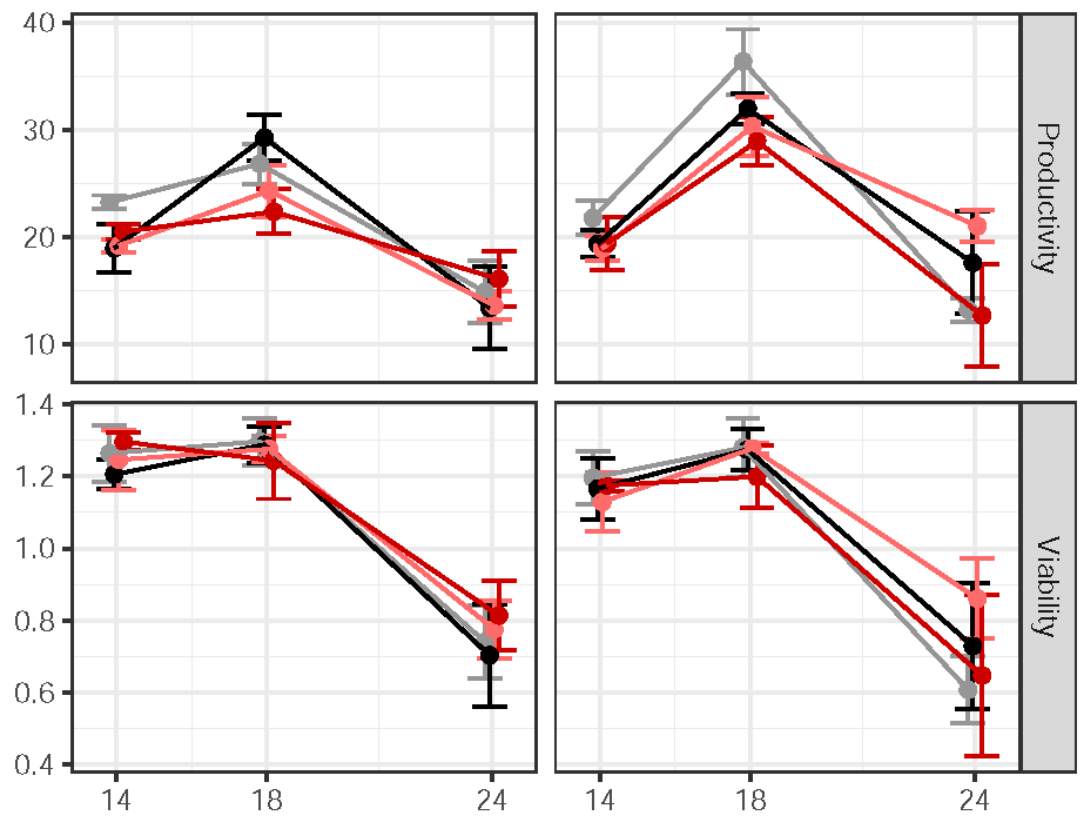

Temperature $\left({ }^{\circ} \mathrm{C}\right)$

718 Figure 3 